\title{
Trapping of Dust Particles into Internal Resonances with the Inner Planets
}

\author{
Rodney S. Gomes \\ Observatório Nacional - Brazil
}

\begin{abstract}
Trapping of dust particles into external resonances with the inner planets is a quite relevant phenomenon, which induces the formation of rings of dust around the planets' orbits, there being observational evidence of the Earth's resonant ring (Dermott et al., 1994). On the other hand, notwithstanding it is a less probable event, capture into internal resonance (diverging orbits) is predicted in numerical integrations of dust particles' orbits subject to Poynting-Robertson drag with one or two disturbing planets. Average theories, which give a good explanation for resonance trapping when the orbits are converging, do not allow capture for diverging orbits. Close approaches with the perturbing planet disallow the application of average theories, and numerical examples show that resonance trapping for internal resonances must have a chaotic origin. As expected from analytical theories, for the case of one disturbing planet, the particle's eccentricity decreases continually after capture and resonance is eventually broken. When two disturbing planets are present, however, there is not necessarily a decrease of the eccentricity, but it may increase, decrease or oscillate, although escape from resonance always occurs anyway.
\end{abstract}

\section{Introduction}

When a dust particle spirals towards the Sun through the action of PoyntingRoberson drag it encounters many mean motion resonances with the inner planets. External resonances are reached first and when the particle succeeds in crossing the highly perturbative region near a planet without suffering any abrupt change in its orbital elements it will also cross the internal resonance region. Because Poynting-Robertson is a dissipative force, external resonances will be associated with converging orbits whereas internal resonances will be associated with diverging orbits. Classical average theories (Henrard, 1982) do not allow resonance trapping for diverging orbits. However, numerical integrations of the complete equations for a dust particle affected by Poynting-Robertson drag in the field of the Sun and one or more perturbing planets will show several trappings into internal resonances with these planets (Marzari and Vanzani,1994). A more detailed numerical analysis of the trapping process shows a chaotic character for these internal resonance trappings, due to moderate close approaches to the perturber. This may explain why average theories are not adequate to explain this kind of trapping. This is also confirmed by the fact that internal 

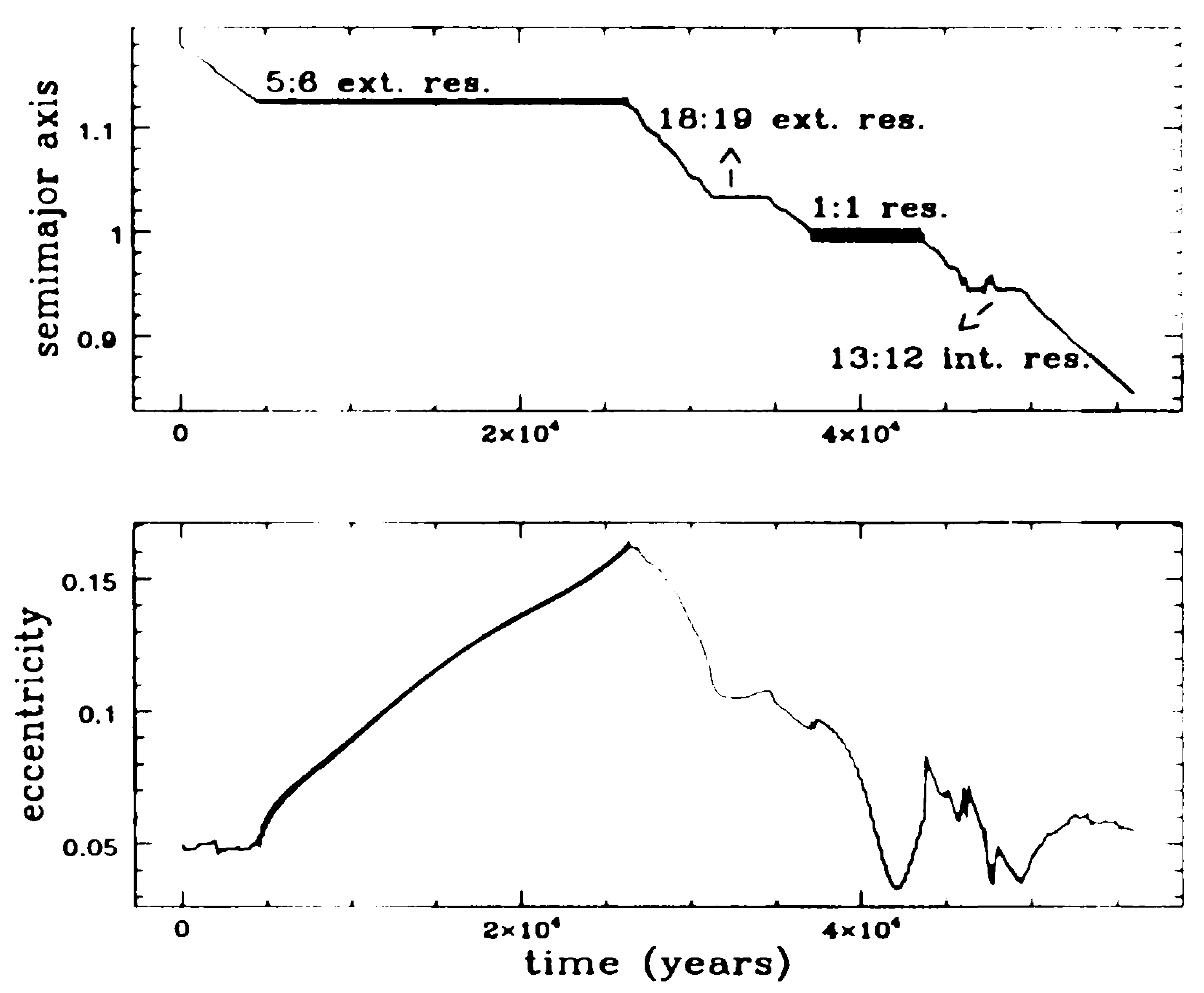

Figure 1. Orbital evolution of a dust particle subject to PoyntingRobertson drag, in the gravitational field of the Sun and the Earth.

resonance trappings occur for the case of high ' $j$ ' resonances, when the particle is close enough to the perturber for close approaches to occur. After trapping into an internal resonance, the eccentricity always start decreasing, in this case confirming analytical theories (Gomes,1995). When there is more than one perturbing planet, this behavior of the eccentricity does not necessarily shows. In this case we can observe a decrease, an increase or an oscillation of the eccentricity. Whenever the eccentricity does not start decreasing after capture, it is noticed that we are in fact dealing with a double resonance, where the particle's mean motion is commensurable with both the external and the internal planets' mean motions. This case may generate less unstable resonance locks in comparison with pure internal resonance locks.

\section{Trapping into Internal Resonances}

Figure 1 shows a typical example of the evolution of a dust grain subject to Poynting-Roberson drag $(\beta=0.01)$ in the gravitational field of the Sun and the Earth. We can notice two external resonance trappings, the $1: 1$ resonance trapping and one internal resonance trapping. The trapping time for an external resonance is considerably longer than the trapping time for an internal resonance. Thus one should not expect an observable proof of capture into internal resonances due to its very unstable character. Nevertheless the dynamics of these trappings is interesting in itself.

Let us consider the variables $P=(\mu a)^{1 / 2}\left[1-\left(1-e^{2}\right)^{1 / 2}\right]$ and $\phi=(j+$ $k) / k \lambda-j / k \lambda_{p}-\varpi$, where $\mu=n^{2} a^{3}$ is a constant, $a$ is the semimajor axis, $e$ is the eccentricity, $\lambda$ is the mean longitude and $\varpi$ is the longitude of the perihelion, all referring to the particle. $\lambda_{p}$ is the mean longitude of the perturbing planet; $j$ and $k$ are integers. In a restricted three-body problem, $P$ and $\phi$ are conjugate canonical variables in a one-degree of freedom averaged Hamiltonian system. Near a resonance this system defines separatrices which are curves that delimit 


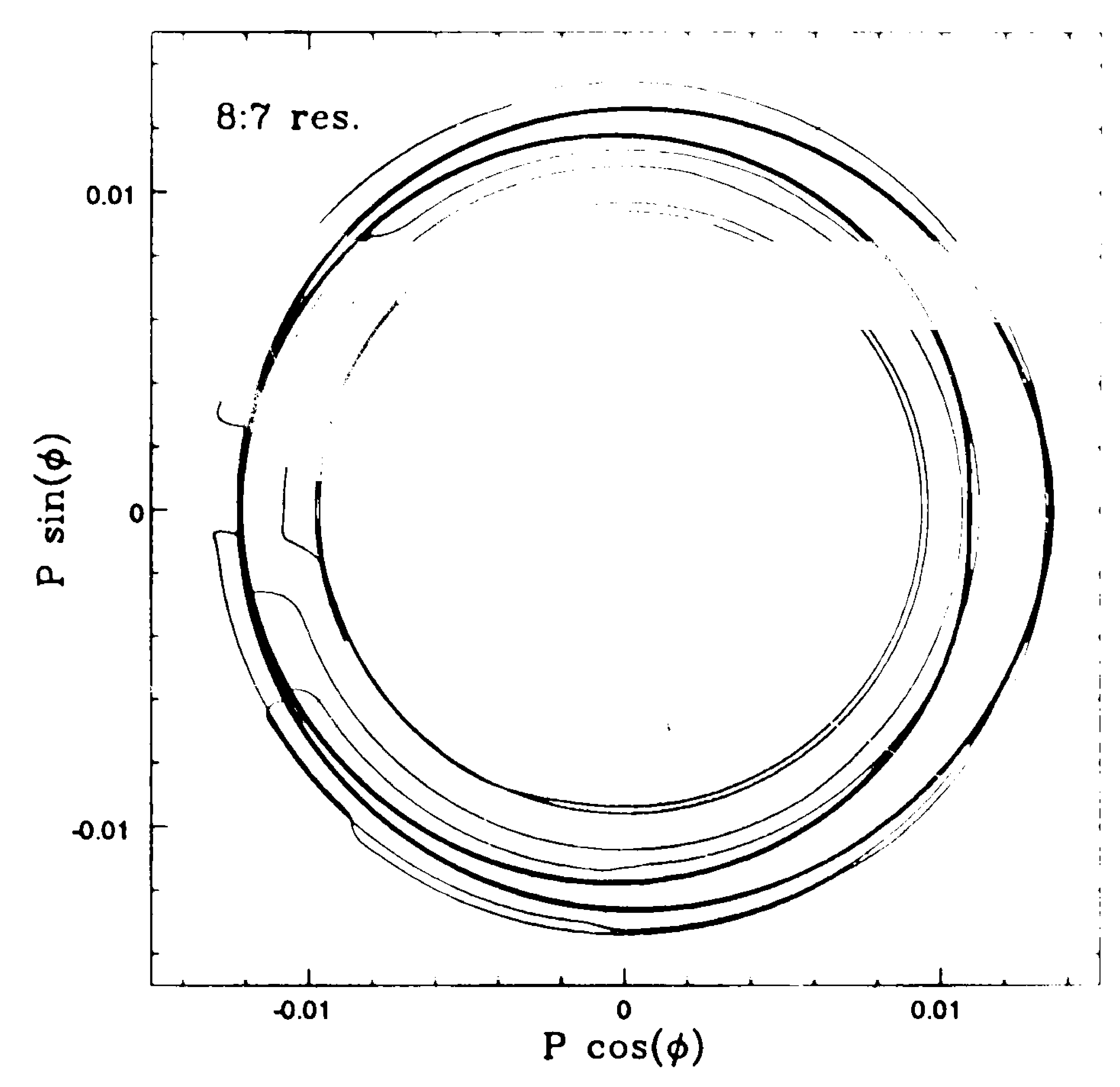

Figure 2. Evolution of the canonical variables $P$ and $\phi$, showing a capture into an internal resonance.

the resonant region. Figure 2 shows an example of these separatrices (the thicker lines), analytically computed from an average time during the evolution of a dust particle's orbit entering a resonance. The thinner line accounts for the variation of the pair $(P, \phi)$ coming from a numerical integration. We notice that the resonant angle $\phi$ starts to librate after its conjugate $P$ suffers some jumps that places the $(P, \phi)$ point into the external circulating zone. These jumps are associated with close approaches to the perturber. The interesting feature here is that the resonant variables hardly stay at the very resonant region (between the two separatrices), but it continually switches from an internal circulation to an external circulation, which in practise has the same effect of a libration (although the libration amplitude here may be higher than expected from an average theory, limited, in this case, by the internal and external separatrices). Being or not captured and staying in resonance depends on the increase and decrease that the variable $P$ suffers when $\phi$ gets near $180^{\circ}$ and this in turn seems to have a chaotic nature.

\section{Double Resonances}

Numerical simulations including two perturbing planets show several examples of resonance trappings in the region between the two planets' orbits. Some are clearly exterior resonance trappings with the innermost planet. On the other hand we find trappings into interior resonances with the outermost planet, with a different behavior for the eccentricity. In fact, when there is more than one perturbing planet, the eccentricity may decrease, increase or oscillate after capture into an internal resonance. The cases not associated with a decrease of the eccentricity after capture are clearly examples of a double resonance, where there is also a mean motion commensurability with the innermost planet. The examples where the eccentricity increases after capture are mostly noticed for the pair Mars-Earth. This usually happens for internal first order resonances 

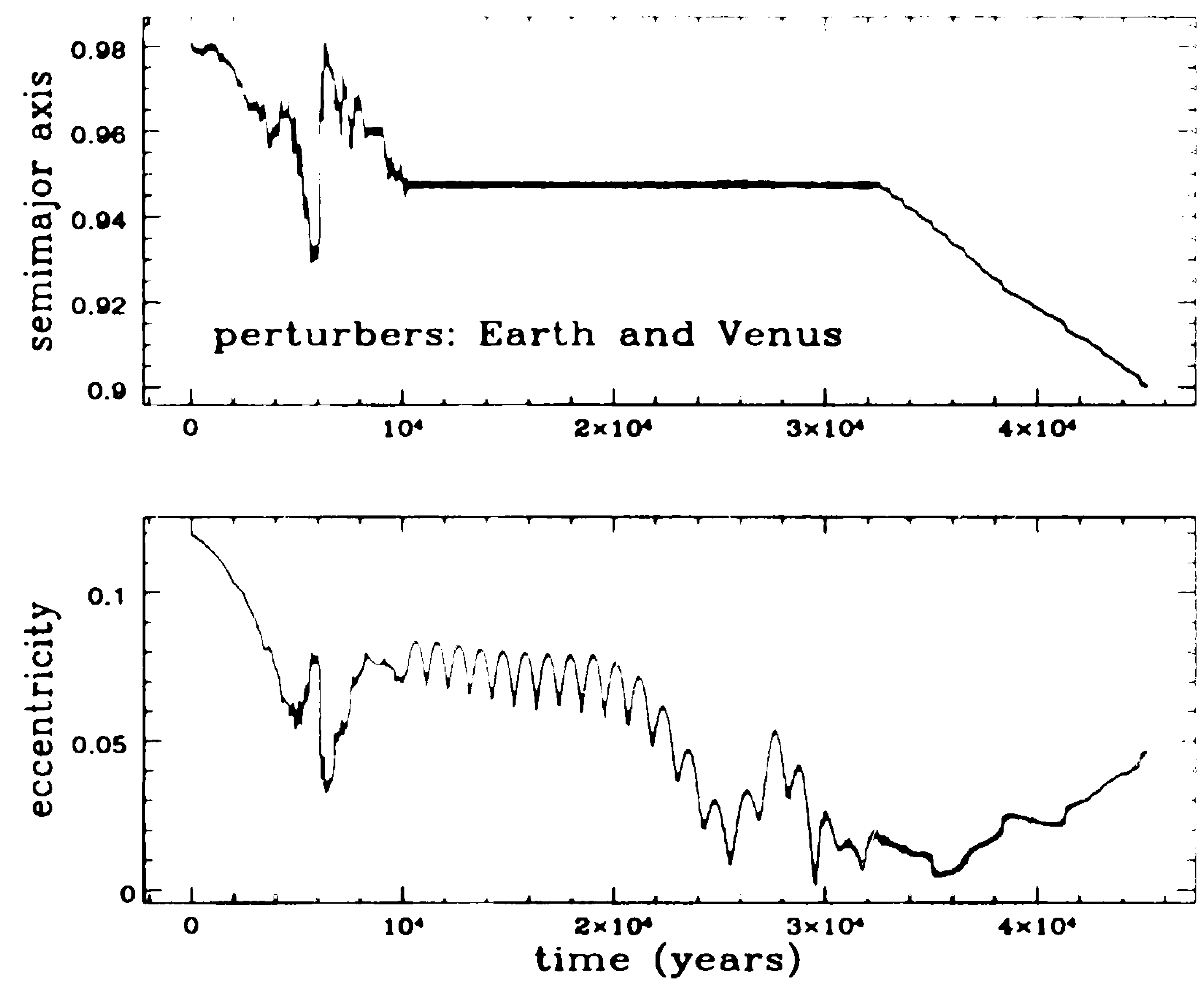

Figure 3. Example of a double resonance lock for the pair Earth-Venus

with Mars and a high order external commensurability with the Earth. One example of double resonance found in numerical integrations was the 30:29 internal resonance with Mars coupled with the 11:20 commensurability with the Earth. Although we should call this a trapping into an internal resonance, because there would be hardly any trapping into such a high order resonance with the Earth, after capture, this external resonance starts to act in such a way that it provokes the increase of the eccentricity which would not otherwise increase by the effect of the internal resonance. The much larger Earth's mass as compared to Mars's must be responsible for this phenomenon.

A final example is shown in figure 3. Here we have another example of a double resonance. It is the 13:12 internal resonance with the Earth and the important 2:3 external resonance with Venus. In this case both planets seem to have important influence in the after-capture eccentricity behavior. The Earth would provoke the eccentricity decrease whereas Venus would try to push it up. The result is an oscillation of the eccentricity during a long time, although in the end it eventually goes down and resonance is broken. This is probably the most remarkable example of double resonance for inner planets' pairs, because it associates a first order (with moderately high $j$ ) internal resonance with the Earth with a first order (low $j$ ) external resonance with Venus.

\section{References}

Dermott,S.F., S.Jayaraman, Y.L.Xu, B.A.S.Gustafson and J.C.Liou 1994, Nature, 369, 719.

Gomes, R.S. 1995, Icarus, 115, 47.

Henrard, J. 1982, Celestial Mechanics, 27, 3.

Marzari, F. and Vanzani, V. 1994, $A \mathcal{B} A$, 283, 275. 